\title{
GÉNERO Y PLANIFICACIÓN URBANA EN LA CONSTRUCCIÓN DE LA AGENDA INTERNACIONAL PARA EL DESARROLLO SOSTENiBle. De Estocolmo 1972 A Quito 2016
}

Gender and Urban Planning in the Construction of the International Agenda for Sustainable Development. From Stockholm 1972 to Quito 2016

\author{
Inés Novella Abril \\ ines.novella.abril@gmail.com \\ Universidad Politécnica de Madrid
}

RESUMEN: La igualdad entre sexos es un requisito fundamental para el desarrollo sostenible que sólo puede garantizarse con la incorporación del mainstreaming de género en las políticas dirigidas a tal efecto. Ante el fenómeno creciente de la urbanización, la dimensión urbana del desarrollo sostenible requiere especial atención, tanto por su transversalidad — porque incluye las tres dimensiones del desarrollo sostenible - como por su impacto — por el efecto motor de las ciudades a escala global—. Avanzar en la incorporación de la perspectiva de género en las políticas de planificación urbana no sólo supone dar respuestas a cambios necesarios en el ámbito local, sino que los beneficios pueden alcanzar la escala global y todos los ejes sobre los que se articula el desarrollo sostenible. Por otro lado, vincular la igualdad entre hombres y mujeres al desarrollo sostenible y aprovechar la inercia de las grandes agendas internacionales, puede ayudar a vencer las importantes resistencias que el género tiene para acceder y consolidarse dentro de la política de planificación urbana. Este texto ahonda en la interrelación entre igualdad, planificación urbana y desarrollo sostenible a través de un recorrido por la evolución conjunta que han experimentado estas tres cuestiones en las principales agendas de las Naciones Unidas, desde Estocolmo 1972 hasta Quito 2016.

Palabras clave: desarrollo sostenible, Nueva Agenda Urbana, urbanismo con perspectiva de género, Agenda 2030. 
ABSTRACT: Gender equality is a fundamental requirement for sustainable development, which can only be achieved by the inclusion of gender mainstreaming in all policies addressing that end. In view of the increasing phenomenon of urbanization, the urban dimension of sustainable development calls for particular attention, given both its transversality, including the three dimensions of sustainability, and its impact, as cities are drivers of change at the global level. Enhancing the gender perspective in urban policies implies not only addressing local requirements, but also having an impact on a global scale and responding to all aspects of sustainable development. In addition, engaging gender equality with sustainable development and harnessing the strength of the major international agendas may contribute to overcome the considerable resistance to gender mainstreaming in urban planning policies. This paper explores the relationship between gender equality, urban planning and sustainable development, covering the co-evolution of these three issues in the most important United Nations international agendas, from Stockholm 1972 to Quito 2016.

KEYWORDS: sustainable development, New Urban Agenda, gender-sensitive urban planning, Agenda 2030.

RESUM: La igualtat entre sexes és un requisit fonamental per al desenvolupament sostenible que només es pot garantir amb la incorporació del mainstreaming de gènere en les polítiques dirigides a aquest efecte. Davant el fenomen creixent de la urbanització, la dimensió urbana del desenvolupament sostenible requereix especial atenció, tant per la seva transversalitat - perquè inclou les tres dimensions del desenvolupament sostenible - com pel seu impacte - per l'efecte motor de les ciutats a escala global-. Avançar en la incorporació de la perspectiva de gènere en les polítiques de planificació urbana no només suposa donar respostes a canvis necessaris en l'àmbit local, sinó que els beneficis poden arribar a l'escala global i tots els eixos sobre els quals s'articula el desenvolupament sostenible. D'altra banda, vincular la igualtat entre homes i dones al desenvolupament sostenible i aprofitar la inèrcia de les grans agendes internacionals, pot ajudar a vèncer les importants resistències que el gènere té per accedir i consolidar-se dins de la política de planificació urbana. Aquest text aprofundeix en la interrelació entre igualtat, planificació urbana i desenvolupament sostenible a través d'un recorregut per l'evolució conjunta 
que han experimentat aquestes tres qüestions en les principals agendes de les Nacions Unides, des d'Estocolm 1972 fins a Quito 2016.

Paraules Clau: desenvolupament sostenible, Nova Agenda Urbana, urbanisme amb perspectiva de gènere, Agenda 2030.

\section{Introducción}

$\mathrm{L}^{\mathrm{a}}$ a Nueva Agenda Urbana (NUA) ${ }^{1}$ es el documento resultante de la Conferencia Hábitat III, celebrada en octubre de 2016 en Quito. La NUA se centra en las acciones que deben llevarse a cabo para garantizar que las zonas urbanas estén planificadas, desarrolladas y gestionadas de manera sostenible a nivel local para que ellas mismas se conviertan en motores de desarrollo sostenible a nivel global. Con este enfoque, la NUA trata de dar respuesta a las dimensiones urbanas de la gran variedad de temáticas que recogen los Objetivos de Desarrollo Sostenible (ODS), que constituyen el plan de acción de la Agenda 2030, la hoja de ruta que rige todo el sistema de las Naciones Unidas en la actualidad. En el plano de las políticas internacionales la idea de sostenibilidad ha pasado de recoger sólo asuntos medioambientales a integrar e interrelacionar prácticamente todos los temas sectoriales. Hoy, el desarrollo sostenible es aquél que combina de manera armónica la sostenibilidad económica, la social y la ambiental, siendo consciente de la interdependencia a corto, medio y largo plazo de estas tres esferas.

La evolución del término desarrollo sostenible hacia un concepto más complejo e integrador no podría entenderse sin la aparición de la perspectiva de género, ni sin el peso que las ciudades han ido ganando en las políticas internacionales en materia de sostenibilidad. El género ha contribuido a detectar y explicar la falta de neutralidad en el desarrollo, las desigualdades y asimetrías (no sólo entre sexos) en el acceso a los recursos, la toma de deci-

1. NUA son las siglas en inglés: New Urban Agenda. El documento completo puede descargarse en: http://habitat3.org/wp-content/uploads/Draft-Outcome-Document-of-Habitat-III-S.pdf 
siones y el impacto de la contaminación; y también ha evidenciado el papel imprescindible de las mujeres en la economía, el mantenimiento del medio ambiente y la gestión de los recursos naturales. La igualdad entre hombres y mujeres se ha ido consolidando como requisito imprescindible para lograr la conservación del medio ambiente y el progreso económico y social. Por este motivo, la perspectiva de género - a su vez herramienta necesaria para garantizar dicha igualdad entre sexos- es imprescindible para el desarrollo sostenible. Por su parte, el crecimiento (tanto demográfico como territorial) de las zonas urbanas ha puesto el foco del desarrollo sostenible en las ciudades, que han pasado de ser consideradas una representación del problema a combatir a entenderse como agentes ineludibles en la implementación de políticas encaminadas hacia el objetivo de la sostenibilidad en su sentido más amplio. No es casualidad que una conferencia centrada en las cuestiones urbanas, como Hábitat III, sea la primera gran conferencia de las Naciones Unidas tras la aprobación de la Agenda 2030 y los oDs. Así pues, en buena medida, el éxito del desarrollo sostenible pasa por atender de manera especial a las áreas urbanas y por garantizar que la perspectiva de género está incorporada transversalmente y de manera sustancial.

En las últimas décadas, en ciudades concretas y de manera intermitente, el género se ha introducido en la planificación y el diseño urbanos, ${ }^{2}$ pero no ha sido nunca una prioridad, no se ha aplicado de manera sustancial, ni se ha incorporado estructuralmente en las políticas urbanas (Sánchez de Madariaga, 2017), la práctica profesional y la formación en materia de urbanismo (Novella, 2016). Es imprescindible que el género llegue a temas con verdadero poder transformador como la movilidad, las infraestructuras, el big data, la gestión de residuos o la política energética; temas, todos ellos, con una fuerte componente urbana. Las resistencias a la incorporación del género en cualquier área política son muy importantes, por eso es necesario un sólido conocimiento en la materia y estrategia en la selección de las medidas. Vincular la igualdad entre hombres y mujeres al desarrollo sostenible puede reforzar la consolidación del género en la planificación urbana, aprovechar

2. El libro Fair Shared Cities editado en 2013 por Inés Sánchez de Madariaga y Marion Roberts, recoge los principales avances en la incorporación del género en las políticas de planificación urbana a escala europea. 
la inercia de las agendas internacionales y expandir su impacto y área de influencia, más allá del diseño del espacio doméstico y los equipamientos de escala vecinal, o la vivienda. ${ }^{3}$ Para ello, es necesario conocer la evolución, casi paralela, que han experimentado el desarrollo de las políticas de desarrollo sostenible, las políticas por la igualdad de oportunidades entre hombres y mujeres y el creciente fenómeno de la urbanización; desde Estocolmo 1972 hasta Quito 2016.

En este artículo hago un recorrido por los principales hitos en la evolución de las políticas de desarrollo sostenible de las Naciones Unidas, deteniéndome en los aspectos directamente relacionados con la igualdad, género o mujeres y niñas, y aquellos relacionados con la planificación de los asentamientos humanos. Mi intención es poner en relevancia que la inclusión de la perspectiva de género en la planificación urbana es clave para la sostenibilidad social, económica y ambiental; no sólo de las propias ciudades y su área de influencia, sino para todo el planeta. Por tanto, el éxito del desarrollo sostenible pasa por lograr una sociedad donde hombres y mujeres gocen de las mismas oportunidades, y por políticas de urbanización y la regeneración urbana capaces de generar asentamientos humanos sostenibles.

\section{La convergencia en el enfoque de las agendas internacionales sobre desarrollo sostenible, igualdad de género y la planificación urbana}

El desarrollo sostenible es actualmente el eje central de las principales agendas internacionales. Naciones Unidas define desarrollo sostenible como «el desarrollo capaz de satisfacer las necesidades del presente sin comprometer la capacidad de las futuras generaciones para satisfacer sus propias necesidades» (Naciones Unidas, 1987). Pero ni éste ha sido siempre el enfoque

3. La vivienda y su entorno urbano de proximidad son temas de gran importancia desde el punto de vista de género, son los temas urbanos donde más iniciativas con perspectiva de género podemos encontrar, generalmente a través de proyectos piloto $\mathrm{y}$ actuaciones concretas. La ciudad de Viena es el principal referente al respecto. En los países en desarrollo se han centrado esfuerzos de manera particular en torno a la seguridad urbana, el programa Safer Cities de onU-Hábitat es el más conocido. 
de la sostenibilidad, ni el desarrollo sostenible ha estado siempre tan presente en la agenda política internacional. Hasta el marco actual que constituyen la Agenda 2030 y los oDs se ha recorrido un camino muy largo. Del mismo modo, la perspectiva de género ha ido ganando peso paulatinamente en todos los temas sectoriales permitiendo elaborar mejores diagnósticos, descubrir nuevas interdependencias y proponer medidas con mejor capacidad de respuesta a las necesidades reales del conjunto de la población. En el caso concreto del desarrollo sostenible, el género ha sido una variable determinante en el enfoque actual de la sostenibilidad, de modo que se trata de dos evoluciones casi paralelas con múltiples interrelaciones que vale la pena estudiar de manera conjunta.

Se considera que el inicio de la política internacional sobre desarrollo sostenible arranca en 1972 con la Conferencia de las Naciones Unidas sobre el Medio Humano. ${ }^{4}$ La interrelación entre la acción del hombre y las cuestiones medioambientales aparece ya en esta conferencia - la primera de la onU sobre medioambiente - que se consigue organizar gracias a las evidencias aportadas por numerosos estudios previos realizados desde el ámbito científico y a la presión de distintos movimientos ciudadanos. Los resultados de esta conferencia incluyen la Declaración de Estocolmo, un Plan de Acción y otras resoluciones y acuerdos como, por ejemplo, establecer el 5 de junio como el Día Internacional del Medio Ambiente. ${ }^{5}$ Los principios recogidos en la Declaración y el Plan de Acción van dirigidos a temas principalmente ambientales y ecológicos, pero ya se relacionan con otros temas como la pobreza, los derechos humanos, la ayuda al desarrollo o el armamento, aunque de una forma un poco vaga y sin establecer de manera clara las interrelaciones entre unos y otros. Hay una referencia en concreto a los asentamientos urbanos, pero orientada a solucionar los problemas derivados de la contaminación. Aunque ya se intuye un llamamiento a atender la cuestión medioambiental desde la unión internacional, el Plan de Acción va dirigido fundamentalmente a los gobiernos nacionales como los principales responsables de la implementa-

4. También conocida como Conferencia de Estocolmo. En algunos casos se considera a ésta como la primera Cumbre de la Tierra.

5. Un informe completo de la Conferencia de Estocolmo, incluyendo la Declaración y el Plan de Acción, puede descargarse en la web de las Naciones Unidas. Link: http:// www.un-documents.net/aconf48-14r1.pdf 
ción de políticas medioambientales. En ninguno de los documentos derivados de la Conferencia de Estocolmo se hace referencia alguna al género, a la igualdad entre sexos o a las mujeres de manera particular.

En los siguientes quince años Naciones Unidas emite tres paquetes de documentos que suponen un avance importantísimo hacia el concepto actual de desarrollo sostenible: La Declaración de Vancouver (1976), la Convención sobre la Eliminación de Todas las Formas de Discriminación contra la Mujer (CEDAw) $^{6}$ (1979) y el Informe Brundtland (1987). En primer lugar, en 1976 se organiza la primera Conferencia de las Naciones Unidas sobre Asentamientos Humanos (Hábitat I) a partir de la cual se crea onU-Hábitat —el Programa de Naciones Unidas para los Asentamientos Humanos - cuya motivación inicial era prevenir y dar solución a los problemas originados por el rápido y descontrolado crecimiento urbano, dirigiéndose especialmente a las ciudades de los países en vías de desarrollo. Sin embargo, el enfoque y los contenidos de la Declaración y el Plan de Vancouver, ${ }^{7}$ van más allá de cuestiones urbanas, y acabarán influyendo en las posteriores políticas de desarrollo de las Naciones Unidas. Estos documentos no sólo reconocen que las condiciones de las zonas urbanas afectan de manera directa al desarrollo humano, social y económico, teniendo graves consecuencias a nivel ambiental, sino que también recogen otros temas sectoriales y una estrategia para la acción internacional que sienta las bases de las siguientes agendas de desarrollo sostenible. En Hábitat I, las cuestiones urbanas se planteaban ya de una manera holística y contemplando la escala mundial, interrelacionando temas políticos, espaciales, sociales, culturales, económicos y medioambientales; aparece incluso la participación ciudadana como uno de los ejes de acción. Sin embargo, el

6. La Convención sobre la Eliminación de Todas las Formas de Discriminación contra la Mujer es un tratado internacional sobre los derechos de las mujeres; fue aprobado por las Naciones Unidas en diciembre de 1979, y entró en vigor en septiembre de 1981. La CEDAW define discriminación como «toda distinción, exclusión o restricción basada en el sexo que tenga por objeto o por resultado menoscabar o anular el reconocimiento, goce o ejercicio por la mujer, independientemente de su estado civil, sobre la base de la igualdad del hombre y la mujer, de los derechos humanos y las libertades fundamentales en las esferas política, económica, social, cultural y civil o en cualquier otra esfera» (Naciones Unidas, 1979).

7. La Declaración y el Plan de Acción de Vancouver están disponibles en la web de ONU-Hábitat. Link: https://unhabitat.org/wp-content/uploads/2014/07/The_Vancouver_Declaration_1976.pdf 
género sigue sin aparecer y las referencias a las mujeres se limitan a que éstas sean consideradas como uno de los grupos vulnerables, y a promover su participación en la actividad política, social y económica, particularmente en la toma de decisiones respecto al planeamiento de los asentamientos humanos, dado el tema central de la conferencia.

La entrada en vigor de la $\mathrm{CEDAW}^{8}$ y su progresiva ratificación entre los países firmantes marcará, precisamente un antes y un después en la presencia y calado de las cuestiones de las mujeres y el género en las distintas agendas internacionales; muy especialmente en lo referente a desarrollo sostenible. La influencia de la CEDAW es apreciable ya en el Informe Brundtland (1987) considerado como el primer documento donde se recoge la definición actual de desarrollo sostenible - que incluirá un mayor número de referencias a las mujeres, y considerará la desigualdad entre sexos como una de las barreras para el desarrollo sostenible. Este informe, también conocido como «Nuestro Futuro Común», fue elaborado por la Comisión Mundial para el Medio Ambiente y el Desarrollo (wCED) ${ }^{9}$ de las Naciones Unidas. Además de tener en cuenta la CEDAw, el Informe Brundtland incorpora también en su texto parte de las propuestas de Vancouver; particularmente, su planteamiento integrador en cuanto a los temas, y su enfoque global como escala de trabajo. A partir de aquí «sostenibilidad»y «desarrollo» serán dos conceptos inseparables en la agenda política internacional, y tanto el género como las cuestiones urbanas irán ganando peso en las políticas de desarrollo sostenible.

El encargo a la Comisión WCED se dividía fundamentalmente en dos partes: primero, realizar un diagnóstico de la situación a escala global y, segundo, elaborar propuestas de acción, innovadoras y realistas para promocionar el desarrollo sostenible. Respecto al primer bloque de trabajo, cabe destacar

8. La CEDAw es un punto de inflexión en el reconocimiento de los derechos de las mujeres a escala internacional. La convención exige a los Estados no sólo que su marco legislativo, judicial y administrativo no discrimine a las mujeres, sino que establezcan medidas para equilibrar los asimétricos roles sociales que hombres y mujeres han tenido tradicionalmente, con el objetivo de alcanzar la igualdad real entre sexos. España ratificó el texto en 1984, comprometiéndose a luchar contra toda forma de discriminación de las mujeres.

9. La Comisión wCED fue creada por la onu en 1983. Gro Harlem Brundtland, Antigua Primera Ministra de Noruega, fue nombra presidenta de la comisión; por ello esta comisión también es conocida como la Comisión Brundtland. 
que, aunque el Informe Brundtland no puede considerarse un documento resultante de un proceso participativo, sí incorpora en el diagnóstico las aportaciones recogidas en una serie de consultas y audiencias públicas realizadas por la Comisión WCED durante casi 3 años, en las que personas y organizaciones de diferentes perfiles procedentes de países de los cinco continentes fueron exponiendo sus preocupaciones y puntos de vista. En cuanto al contenido del informe, los temas urbanos y el género aparecen en mayor medida y más interrelacionados que en la Declaración de Vancouver, pero todavía no de manera sustantiva. Por ejemplo, el crecimiento de las ciudades se considera principalmente como un reto; sobre todo, en el caso de las ciudades de países en desarrollo. Pero, sí se hace alguna referencia a los problemas de las ciudades de países con rentas altas, donde también se apunta al potencial de las ciudades como promotoras de desarrollo sostenible. ${ }^{10}$ En cualquier caso, el informe dedica un capítulo completo a los temas urbanos dentro del cual se comienzan a interrelacionar temas sectoriales tratados en otros apartados del documento con el contexto urbano y se reconoce el potencial que las autoridades locales tienen para contribuir al desarrollo sostenible.

Respecto a cuestiones relacionadas con las mujeres, el género y la igualdad entre sexos, el Informe Brundtland - con una clara influencia de la Convención CEDAW - supone un salto cualitativo respecto a documentos anteriores; pero sigue sin haber un planteamiento transversal. En el Informe Brundtland hay numerosas referencias a la desigualdad entre hombres y mujeres como barrera para el desarrollo sostenible, pero la mayoría de ellas se relacionan con salud reproductiva y sexual, fundamentalmente con el objetivo de frenar el crecimiento descontrolado de la población mundial. Es una visión todavía muy sesgada de la problemática, que descarga exclusivamente en la mujer la responsabilidad reproductiva, y alrededor de la cual se conectan otros temas como el acceso a la educación y a la toma de decisiones. En la línea de todo el informe, las cuestiones de género están principalmente enfocadas a los países empobrecidos y al contexto de la cooperación al desarrollo donde se reconoce el papel protagonista de las mujeres en la seguridad alimentaria, como principales productoras, procesadoras y comerciantes de alimentos y

10. Ver capítulo 9, pp. 202-203 (Naciones Unidas, 1987). 
se insta a trabajar sobre aspectos como la igualdad en la titularidad de la tierra u otorgar cierta prioridad a las mujeres en la concesión de recursos y financiación. No hay mención al género en cuestiones tan relevantes como el papel de la economía internacional, la movilidad, o la política energética. Sin embargo, cuando se plantean las estrategias para el desarrollo sostenible, se reconoce que el contexto urbano puede impulsar el desarrollo sostenible ya que facilita la incorporación de las mujeres a la toma de decisiones, al empleo y a la educación. Aunque es interesante y muy pionera esta interrelación explícita ${ }^{11}$ entre desarrollo sostenible, género y cuestiones urbanas, el Informe Brundtland sigue teniendo una visión poco amplia y muy centrada en temas reproductivos. La mejora de la situación de las mujeres gracias a las oportunidades que ofrecen las ciudades se considera que tiene un impacto positivo en el desarrollo sostenible, fundamentalmente porque el mayor acceso a la educación sexual y reproductiva que asegura el contexto urbano permite establecer un cierto control de la natalidad y, por extensión, puede contribuir a establecer unos niveles de población mundial sostenibles social, económica y ambientalmente.

Aparte de definir el concepto actual de desarrollo sostenible, el Informe Brundtland es un documento importantísimo porque deja claro que es preciso pasar a la acción y hace un llamamiento tanto a los responsables políticos como al conjunto de la sociedad, para que se implique activamente en un cambio de modelo de desarrollo. La transversalidad que se asocia a esta idea de desarrollo sostenible requiere modificar las estructuras institucionales para que cada ámbito de la política integre la sostenibilidad en su agenda. Naciones Unidas introdujo las premisas del Informe Brundtland en todos los programas y convocó una nueva Conferencia Sobre Medio Ambiente y Desarrollo para reorientar la agenda política internacional a partir de las conclusiones de este documento. Esta conferencia es la conocida como Cumbre de la Tierra, o Conferencia de Río, celebrada en Río de Janeiro en junio de 1992.

La Conferencia de Río fue la cumbre más grande organizada por la onU en materia de sostenibilidad. El objetivo principal del encuentro era definir una agenda concreta de impacto global pero dirigida principalmente a los

11. Ver artículo 51, p. 51 (Naciones Unidas, 1987). 
Estados para que éstos redirigiesen los modelos de desarrollo existentes hacia otros más sostenibles a nivel económico, social y ambiental. La relevancia de la Cumbre de Río se debe, por un lado, a su repercusión mediática a nivel global, lo que haría posible acercar la idea de desarrollo sostenible al público mayoritario por primera vez; algo fundamental para conseguir el cambio en los comportamientos individuales que proponía el Informe Brundtland. Por otro lado, a los documentos y acuerdos resultantes, que hacen de esta cumbre otro hito en la evolución de las políticas de desarrollo sostenible. De Río surgen documentos tan importantes como la Agenda 21 y la Declaración de Río sobre Medio Ambiente y Desarrollo, ${ }^{12}$ pero también acuerdos vinculantes como la Convención Marco de las Naciones Unidas sobre el Cambio Climático. $^{13}$

El documento más relevante de la Conferencia de Río en el tema que nos ocupa es la Agenda 21, ${ }^{14}$ que constituye el Plan de Acción derivado de esta cumbre. La Agenda 21 va dirigida a la propia onU, a otros organismos multilaterales, a los Estados y a otros grupos de interés ${ }^{15}$ a los que se les insta a establecer, en su ámbito de aplicación y en la medida de sus posibilidades, su propia estrategia a favor del desarrollo sostenible. El documento se divide en cuatro bloques: el primero, que aborda la dimensión social y económica del desarrollo sostenible; el segundo, que trata los temas de conservación y gestión de los recursos; el tercero, que va dedicado al fortalecimiento de los

12. El Principio 20 de la Declaración de Río es la única mención a cuestiones relacionadas con las mujeres. En él se reconoce a las mujeres como agentes fundamentales en la ordenación del medioambiente y el desarrollo, por lo que se considera imprescindible su plena participación para la consecución del desarrollo sostenible. El texto completo de la Declaración de Río está disponible en: http://www.unesco.org/education/pdf/ RIO S.PDF

13. La Convención Marco de las Naciones Unidas sobre el Cambio Climático (UNFCCC, por sus siglas en inglés) incluirá más adelante acuerdos como el Protocolo de Kioto (1997) y el Acuerdo de París (2015). A raíz de la Cumbre de la Tierra también se creó la Comisión de las Naciones Unidas para el Desarrollo Sostenible.

14. El término «Agenda 21» es el más empleado; sin embargo, se tradujo al castellano originalmente como «Programa 21», y así es posible encontrarlo en algunos documentos. El texto completo de la Agenda 21 está disponible en: http://www.un.org/ spanish/esa/sustdev/agenda21/agenda21 toc.htm

15. Estos grupos de interés aparecen en la versión española del documento como «Grupos Principales», donde no sólo se incluyen determinados colectivos por su vulnerabilidad (infancia, mujeres, poblaciones indígenas, etc.), sino también aquellos que son relevantes por su potencial y su capacidad de impacto en la implementación de las medidas que la Agenda 21 propone. Por ejemplo, la comunidad científica y las oNG. 
distintos grupos principales; y el cuarto, que aborda los medios de implementación. En la Agenda 21, que hereda el espíritu del Informe Brundtland, se incluyen ya la práctica totalidad de los temas que hoy constituyen los 17 Objetivos de Desarrollo Sostenible. La principal aportación de la Agenda 21 es ofrecer un catálogo de recomendaciones concretas que cubren no sólo esa gran variedad de temáticas, sino que van dirigidas y adaptadas a actores muy distintos. De alguna manera, la Agenda 21 enumera las tareas y distribuye las responsabilidades, para facilitar su ejecución y también establecer mecanismos de control y evaluación.

Las cuestiones relacionadas con los asentamientos humanos se desarrollan en dos capítulos. El capítulo 7 - uno de los más extensos del documentoaborda temas sectoriales de la planificación urbana (vivienda, ordenación del suelo, infraestructuras, energía, movilidad), así como algunas cuestiones como la mejora de la administración local, la previsión frente a los desastres naturales y el fomento de la sostenibilidad en el sector de la construcción. El capítulo 28 , dirigido a las administraciones locales, ${ }^{16}$ reconoce la dimensión urbana de muchos de los temas que atañen al desarrollo sostenible $\mathrm{y}$, por tanto, la importancia de que las ciudades participen activamente en la implementación de las medidas propuestas en la Agenda 21. En este capítulo es también donde se insta a los gobiernos locales a elaborar su propio plan de acción para el desarrollo sostenible, la conocida como Agenda 21 Local. La dimensión urbana del desarrollo sostenible está presente en otros capítulos, sobre todo aquéllos que abordan la gestión del agua dulce y el saneamiento (capítulos 18 y 20).

En cuanto a cuestiones de género e igualdad entre sexos la Agenda 21 es mucho más transversal que el Informe Brundtland. Además de considerar a las mujeres y niñas como uno de los grupos principales y dedicarles en exclusiva un capítulo dentro del bloque 3 , hay referencias explícitas a las mujeres como grupo vulnerable y llamadas a garantizar su plena participación en la práctica totalidad de los capítulos sobre los temas sustantivos del desarrollo sostenible

16. En la Agenda 21, los gobiernos locales constituyen un grupo principal. Aunque se traten de administraciones públicas, en las negociaciones y procesos participativos para las agendas de desarrollo sostenible, las Naciones Unidas consideran a los ayuntamientos como parte de la sociedad civil. 
(bloques 1 y 2), y también en los capítulos sobre implementación (bloque 4). Esto supone que, aunque sigue habiendo mayor presencia de estas cuestiones en campos como la pobreza, la salud sexual y reproductiva o la educación, también comienzan a aparecer en ámbitos como la conservación medioambiental, la deforestación, el agua, los patrones de consumo, la gobernanza, etc. Hay tres cuestiones en la Agenda 21 que indican un avance en la transversalización de la perspectiva de género en el desarrollo sostenible: reconocer la necesidad de crear bases de datos segregados por sexos (capítulos 5, 8 y 12), de incorporar a más mujeres en carreras técnicas para que contribuyan como profesionales en las diferentes dimensiones del desarrollo sostenible (capítulo 35 ), y de introducir la perspectiva de género en los diagnósticos (capítulo 24), en los avances tecnológicos (capítulo 34) y en los sistemas educativos (capítulo 36). Aunque la Agenda 21 no ha tenido el grado de implementación que se esperaba, puede considerarse un éxito por la influencia que ha tenido en las siguientes agendas sobre desarrollo, y en el hecho de haber acercado la escala global del problema a la política y la realidad local.

De nuevo, entre 1995 y 1996, tienen lugar dos conferencias internacionales — una en el ámbito de la igualdad y otra en el de áreas urbanas - que son de relevancia en la evolución de las políticas internacionales de desarrollo sostenible. En 1995 se celebró en Pekín la Cuarta Conferencia Mundial sobre la Mujer, ${ }^{17}$ que marca otro hito en la política internacional por los derechos de las mujeres. De esta conferencia surge la Declaración de Pekín y su plan de acción conocido como la Plataforma de Acción de Pekín. Estos documentos recogen propuestas de la Agenda 21 en cuanto a parte de los contenidos (remarcando el papel de las mujeres en la sostenibilidad del planeta) y en cuanto al planteamiento de establecer áreas prioritarios de acción y objetivos. La propuesta más transformadora de Pekín $95^{18}$ fue cambiar el foco de la problemática desde «las mujeres» al «género», lo que implica que la desigualdad entre hombres y mujeres es un tema transversal que afecta a todos

17. Las Conferencias Mundiales sobre la Mujer reúnen a representantes de los Estados para abordar la desigualdad entre hombres y mujeres, proponer medidas y estrategias, etc. Las tres anteriores Conferencias Mundiales sobre la Mujer se habían celebrado en Méjico (1975), Copenhague (1980) y Nairobi (1985).

18. Los documentos resultantes de la Conferencia de Pekín están disponibles en: http:// www.un.org/womenwatch/daw/beijing/pdf/BDPfA\%20S.pdf 
los ámbitos de la sociedad y que sólo abordándose desde la perspectiva de género es posible generar los cambios necesarios para alcanzar la igualdad. La aplicación de la perspectiva de género en las políticas se denomina transversalidad o mainstreaming de género que supone integrar la perspectiva de género en todas las áreas de la política, a todos los niveles y en cada fase del proceso de desarrollo de la política (diseño, implementación, seguimiento y evaluación). Este enfoque de género ha influido en el desarrollo de las políticas más avanzadas a favor de la igualdad entre sexos, ${ }^{19}$ incluidas las agendas internacionales de desarrollo sostenible.

Por su lado, en 1996 onU-Hábitat organizó en Estambul la 2a Conferencia sobre Asentamientos Humanos (Hábitat II), ${ }^{20}$ dando respuesta así a la Agenda 21 en sus indicaciones sobre la dimensión urbana del desarrollo sostenible y, en especial, para redirigir el fenómeno de la urbanización hacia un proceso sostenible. En cuanto a las áreas de acción y los temas propios de las políticas urbanas, Hábitat II sigue la línea de Hábitat I, considerablemente enfocada hacia los países en desarrollo y la cobertura de necesidades básicas como la vivienda, la accesibilidad a los servicios urbanos básicos, el empoderamiento de las administraciones locales y la especial atención a grupos vulnerables, entre los que se encuentran las mujeres. Precisamente es en la inclusión de la perspectiva de género donde Hábitat II da un salto cualitativo respecto a Hábitat I. No sólo la igualdad entre hombres y mujeres es uno de los siete compromisos de la Agenda Hábitat (art. 46), sino que se reconoce que el mecanismo principal para conseguirlo es la introducción del mainstreaming de género en la política urbana, incorporando así las indicaciones de Pekín 1995.

En septiembre de 2000 la ONU organizó en su sede de Nueva York la Cumbre del Milenio, congregando a los líderes de los Estados miembros para discutir cuál debía ser el papel de las Naciones Unidas en el siglo XXI y definir sus líneas principales de trabajo de toda su estructura. En la Declaración del Milenio, el documento resultante de la cumbre, se ratifica la Carta

19. Como por ejemplo el Tratado de Ámsterdam a escala europea, la Ley Orgánica de Igualdad 3/2007 en el ámbito español, etc.

20. La Declaración de Estambul y su plan de acción (Agenda Hábitat) están disponibles en: http://www.un-documents.net/hab-ag.htm 
de las Naciones Unidas ${ }^{21}$ y se reafirma el convencimiento por parte de los Estados de que la onU es indispensable también en el nuevo contexto global para lograr un mundo más pacífico, más próspero y más justo. Ahora bien, también se reconoce que la globalización ha sido una fuerza transformadora con muchas asimetrías entre países y regiones del mundo, por lo que la nueva agenda debería contener políticas internacionales orientadas a reducir tales desigualdades. Esa agenda la constituyen los ocho Objetivos de Desarrollo del Milenio (ODM) (fig.1) que fueron aprobados en 2001, con un plazo de 15 años para su cumplimiento. En algunos aspectos los ODM son menos ambiciosos que las agendas y acuerdos que los preceden, incluso que la propia declaración de la que parten, ya que ésta apela y ratifica programas como la Agenda 21 o el Protocolo de Kyoto, entre otros. Los oDM vuelven a centrar la agenda de desarrollo de la ONU en torno a los países en desarrollo y a objetivos básicos como la reducción de la pobreza y la protección de los grupos más vulnerables, pero no consiguen integrar bien cuestiones algo más novedosas como el cambio climático o el proceso de urbanización global. De hecho, las dos únicas referencias directas a las cuestiones urbanas están incluidas en el ODM7 («Garantizar la sostenibilidad del medio ambiente») y abordan el acceso a servicios básicos de agua potable y saneamiento, y a mejorar las condiciones de vida en los barrios marginales. Aunque la igualdad entre sexos sí tiene una mayor presencia y constituye en sí dos Objetivos (ODM 3, «Promover la igualdad entre los géneros y la autonomía de la mujer», y oDM 5, «Mejorar la salud materna»), el enfoque es muy poco evolucionado, sin integrar el género y la visión transversal propuesta en Pekín 1995 y volviendo a centrar la atención en el acceso a la educación, la salud sexual y reproductiva, y a la presencia de más mujeres en los parlamentos; es decir, exactamente los mismos parámetros que recogía ya el Informe Brundtland en 1987.

Los ODM fueron criticados por haberse aprobado sin un proceso de participación, especialmente por la falta de negociación con los países empobrecidos, que son los principales destinatarios del programa. También se consideró limitante el enfoque vertical y la falta de interrelación entre los distintos ODM. Pero, sin embargo, hay aspectos de los oDM que han sido valorados

21. La Carta de las Naciones Unidas es el documento constitucional de la ONU. 
positivamente; por ejemplo, la sencillez y claridad de los objetivos y sus indicadores ha facilitado su medición y evaluación, pero también la difusión de los objetivos más allá de la población familiarizada con este tipo de agendas internacionales. Los ODM han contribuido a la concienciación y participación de la población en general y a que ésta, al estar informada e involucrada, exija más rendición de cuentas a los responsables políticos. Pasado el plazo de 15 años, ${ }^{22}$ se considera que los oDM han sido especialmente efectivos en la reducción de la mortalidad infantil y el acceso a la educación primaria, al agua potable y los tratamientos contra algunas enfermedades como el SIDA. Por el contrario, los objetivos han fallado en la reducción de la mortalidad materna, la reducción de las emisiones de gas y la reducción del empobrecimiento en algunas regiones clave (especialmente el sureste asiático). Respecto a estos dos últimos aspectos, es claro que la falta de buenos resultados se debe en buena medida a la ausencia de los países de rentas altas en el enfoque de los objetivos, y a no prever el impacto de la aceleración del fenómeno de la urbanización. Pero, en general, la onU considera satisfactorios los resultados obtenidos por los ODM.

Dentro del plazo de los 15 años establecidos para el cumplimiento de los oDM, se celebraron las Cumbres Río+10 (Johannesburgo, 2002) y Río+20 (Río de Janeiro, 2012) ${ }^{23}$ con el propósito de revisar y evaluar la implementación de la Agenda 21 y acordar nuevas iniciativas para avanzar en el desarrollo sostenible. Es a partir de estas dos conferencias, y tras los primeros resultados de los oDM, cuando se comienza a establecer una convergencia oficial entre la línea de trabajo general de la onU, que en ese momento había sido decidida en la Cumbre del Milenio y se estaba implementando a través de los ODM, con las agendas resultantes de las grandes conferencias sobre el desarrollo sostenible,

22. El análisis de resultados de los Objetivos del Milenio puede consultarse en la web de Naciones Unidas. Link: http://www.un.org/millenniumgoals/

23. Los nombres oficiales de Río+10 y Río+20 son respectivamente: Cumbre Mundial sobre Desarrollo Sostenible (wsSD-World Summit on Sustainable Development), y Conferencia de Desarrollo Sostenible de las Naciones Unidas (UNCSD-United Nations Conference on Sustainable Development). En 1997 se celebró en Nueva York la conferencia Río+5 con el mismo propósito de evaluar el avance de las políticas de desarrollo sostenible tras la cumbre de Río en 1992. Se trata de una conferencia menor, cuyos acuerdos y documentos resultantes son de menor alcance respecto a Río+10 o Río+20, y sin avances sustanciales en el tema que nos ocupa. La resolución de Río+5 está disponible en: http://www.un.org/es/comun/docs/?symbol=A/RES/S-19/2 
cuyo documento más avanzado en ese momento era la Agenda 21, surgida a partir de la Cumbre de la Tierra de 1992.

Respecto al género y la igualdad de oportunidades, se aprecia en el Plan de Acción ${ }^{24}$ de Río+10 la influencia de la Conferencia de Pekín. Las políticas dirigidas a las mujeres se combinan con el mainstreaming de género que aparece ya en gran parte de las áreas de acción, lo que supone un enfoque bastante más avanzado que permite establecer interrelaciones entre unas y otras de mucho interés. Por ejemplo, en el objetivo para la erradicación de la pobreza (un asunto incluido en el área social y económica) se alude a la necesidad de prestar especial atención en temas de habitabilidad (un tema de planificación urbana) a las familias monoparentales (un tema con una gran componente de género). ${ }^{25}$ Respecto a las políticas urbanas, el principal avance se da en un sentido similar al género: la temática se consolida como relevante y se vuelve más transversal. Por ejemplo, en el apartado sobre alternativas a los patrones de consumo y producción, se establecen relaciones entre el modelo de planeamiento urbano con ciertas pautas de consumo energético y de los recursos naturales; se insta, entre otras cosas, a limitar la dispersión urbana y a elaborar planes integrales de transporte a todas las escalas, entendiendo la planificación y la gestión de la movilidad como un aspecto relevante para el desarrollo sostenible. En términos generales, el documento principal de Río+20, «El futuro que queremos», es una continuación de la Agenda 21 y de Río+10; pero es en esta conferencia donde Naciones Unidas inicia el proceso para la elaboración de la que será su hoja de ruta a partir de 2015, una vez el plazo de los odm venza. En Río+20 Naciones Unidas creo el Grupo de Trabajo Abierto para el desarrollo de la Agenda Post2015 que, basándose en las pautas de la Agenda 21 y Río+10, debía también recoger las principales cumbres internacionales en temas económicos, sociales y ambientales. Es decir, aglutinar las tres dimensiones del desarrollo sostenible y sintetizar los principales acuerdos internacionales.

24. Los documentos derivados de Río+10 son la Declaración de Johannesburgo, y su Plan de Acción. Los documentos pueden encontrarse en: http://www.un.org/es/comun/ docs/?symbol=A/CONF.199/20

25. Las familias monoparentales están muy mayoritariamente encabezadas por una mujer. Son uno de los colectivos más desfavorecidos en temas urbanos desde la perspectiva de género (Bosch, 2017). 
La propuesta elevada a la Asamblea General ${ }^{26}$ fue el resultado de un largo proceso de negociación, de carácter multilateral, con consultas nacionales y por temas, e incluía ya un listado con 17 Objetivos de Desarrollo Sostenible (oDs). Este texto fue negociado por los Estados miembros hasta acordar el texto definitivo «Transformando nuestro mundo: la Agenda 2030 para el Desarrollo Sostenible» ${ }^{27}$ en agosto de 2015, que fue finalmente adoptado durante la Cumbre de Nueva York un mes más tarde. Los 17 oDs son el Plan de Acción de la Agenda 2030 y plantean diferencias significativas con los ODM. Se entiende que el trabajo de toda la estructura de la onu gira en torno al desarrollo sostenible, y sus tres dimensiones; el enfoque de los oDs es universal, dirigiéndose tanto a países de rentas altas como a países empobrecidos $\mathrm{y}$, aunque cada objetivo tiene metas concretas y específicas, todos ellos tratan de establecer interrelaciones. Los oDs surgen de un proceso más participativo que los ODM, tanto en la propia definición de los mismos y sus temáticas como en los medios de implementación —especialmente en la movilización de la financiación - y el sistema de control y evaluación, que se trazaron de manera previa.

La igualdad de género constituye el oDs5 y las áreas urbanas el oDs11; son, pues, dos cuestiones prioritarias en la Agenda 2030. El género está implícito en todos los oDs (ONU-Mujeres, 2016), porque la igualdad entre sexos está consolidada como uno de los requisitos básicos para del desarrollo sostenible. Sin embargo, aunque las cuestiones relacionadas con los asentamientos humanos han experimentado una creciente relevancia dentro de las agendas internacionales, la dimensión urbana de los distintos ejes temáticos asociados al desarrollo sostenible en general, y de cada uno de los oDs en particular, está todavía pendiente de desarrollarse políticamente. Precisamente con esta intención ONU-Hábitat convocó la Conferencia Habitat III en octubre de 2016, de la cual surge la Nueva Agenda Urbana (NUA) cuyo objetivo general es desarrollar el oDs11 sobre ciudades sostenibles. La NUA hace numerosas referencias a cuestiones de género e igualdad, pero todavía muy en la línea de considerar a las mujeres

26. La Asamblea General de las Naciones Unidas está formada por los Gobiernos de los Estados miembros, son quienes aprueban en última instancia las agendas y documentos finales.

27. El documento está disponible en: http://www.un.org/ga/search/view_doc.asp?sym$\mathrm{bol}=\mathrm{A} / 70 / \mathrm{L} .1 \&$ Lang $=\mathrm{S}$ 
como colectivo vulnerable, especialmente sensible a la falta de planificación y sostenibilidad de las áreas urbanas. Pero, sí se aprecian algunos planteamientos transformadores desde el punto de vista de género, relacionados fundamentalmente con la innegable dimensión urbana del derecho a la propiedad del suelo, la seguridad en el espacio público y las oportunidades que ofrece la economía informal asociada a las actividades del cuidado (Moser, 2016).

La igualdad entre sexos es un requisito fundamental para el desarrollo sostenible que sólo puede garantizarse con la incorporación del mainstreaming de género en las políticas dirigidas a tal efecto. Pero, en un planeta cada vez más urbanizado, donde las ciudades aglutinan las oportunidades económicas y de desarrollo, los grandes desafíos ambientales y la cada vez mayor población, ${ }^{28}$ la dimensión urbana del desarrollo sostenible requiere especial atención, tanto por su transversalidad - porque se relaciona con las tres dimensiones del desarrollo sostenible - como por su impacto - por el efecto motor que tienen las ciudades a escala global—. Por este motivo, avanzar en la incorporación de la perspectiva de género en las políticas de planificación urbana no sólo supone dar respuestas a cambios necesarios en el ámbito local, sino que los beneficios pueden alcanzar la escala global y todos los ejes sobre los que se articula el desarrollo sostenible.

\section{Referencias}

Bosch, J. (2017). «Políticas de vivienda con perspectiva de género: un análisis desde la crisis». En Serrano, B., Mateo, C. y Rubio, A. (Ed.), Género y Política Urbana. Arquitectura y Urbanismo desde la perspectiva de género (pp. 81-112). Valencia: Instituto Valenciano de la Edificación.

Emakunde (1998). ¿Qué ha supuesto la Conferencia de Pekín para las Mujeres? Bilbao: Instituto Vasco de la Mujer.

Fainstein, S. S. \& Servon, L. J. (2005). Gender and Planning. A Reader. New Jerey/London: New Brunswick/Rutgers University Press.

28. En el año 2017 la población mundial residente en áreas urbanas supera el 50\%. Se espera que en 2030 la población urbana alcance el 60\%. Las ciudades generan en torno al $70 \%$ del PIB mundial, pero también la mitad de los gases de efecto invernadero (ONU-Hábitat, 2016). 
Moser, C. (2016). «Gender Transformation in a New Global Urban Agenda: Challenges for Habitat III and Beyond». Environment and Urbanization, vol 29 (1), 221-236. DOI: 10.1177/0956247816662573

Naciones Unidas (1972). Report of the United Nations Conference on the Human Environment. Recuperado de http://www.un-documents. net/aconf48-14r1.pdf

- (1976). The Vancouver Declaration on Human Settlements. Recuperado de https://unhabitat.org/wpcontent/uploads/2014/07/ The_Vancouver_Declaration_1976.pdf

- (1979). Convención sobre la eliminación de todas las formas de discriminación contra la mujer. Recuperado de http://www.un.org/womenwatch/daw/cedaw/text/sconvention.htm

- (1987). Report of the World Commission on Environment and Development: Our Common Future. Recuperado de http://www.un-documents.net/our-common-future.pdf

— (1992). Agenda 21. Recuperado de https://sustainabledevelopment.un.org/ content/documents/Agenda21.pdf

- (1995). Declaración y Plataforma de Acción de Beijing. Recuperado de http://www.un.org/womenwatch/daw/beijing/pdf/BDPfA\%20S.pdf

- (2000). United Nations Millennium Declaration. Recuperado de http:// www.un.org/millennium/declaration/ares552e.pdf

- (2002). Informe de la Cumbre Mundial sobre el Desarrollo Sostenible. Disponible en: http://www.un.org/es/comun/docs/?symbol=A/CONF.199/20

- (2015). Transformar nuestro mundo: la Agenda 2030 para el Desarrollo Sostenible. Recuperado de http://www.un.org/ga/search/view_doc. asp?symbol=A/70/L.1\&Lang=S

- (2016). Nueva Agenda Urbana. Declaración de Quito sobre Ciudades y Asentamientos Humanos Sostenibles para Todos. Recuperado de http://habitat3.org/wp-content/uploads/Draft-Outcome-Document-of-Habitat-III-S.pdf

Novella Abril, I. (2016). «Reconocimiento en la arquitectura y el urbanismo. Reconfigurar la profesión para la Nueva Agenda Urbana». Territorio della Ricerca su Insedimenti e Ambiente. Vol 9 (2), 39-56. DOI: $10.6092 / 2281-4574 / 5054$

Onu-hábitat (1996). The Habitat Agenda. Istanbul Declaration on Human Settlements. Recuperado de http://www.un-documents.net/hab-ag.htm

- (2012). Gender Issue Guide: Urban Planning and Design. Nairobi: ONUHábitat. 
- (2016). Urbanization and Development: Emerging Futures. World Cities Report 2016. Nairobi: onU-Hábitat.

Onu-Mujeres (2016). Women and Sustainable Development Goals. Nairobi: UN Women Eastern and Southern Africa Regional Office.

Reeves, D., Parfitt, B. \& Archer, C. (2012). Gender in Urban Planning. Issues and trends. Nairobi: ONU-Hábitat.

Sánchez de Madariaga, I., \& Roberts, M. (eds.) (2013). Fair Shared Cities. The impact of gender planning in Europe. Aldershot-Nueva York: Ashgate.

- (2017). «Una nueva generación de políticas para la igualdad efectiva de género en el urbanismo. De la Nueva Agenda Urbana a las Directrices de Ordenación del Territorio Vasco». En Serrano, B., Mateo, C. y Rubio, A. (Ed.), Género y Política Urbana. Arquitectura y Urbanismo desde la perspectiva de género (pp. 113-133). Valencia: Instituto Valenciano de la Edificación.

UndP (2012). Powerful Synergies. Gender Equality, Economic Development and Environmental Sustainability. New York: United Nations Development Programme. 
